\title{
The Relationship between Incorrect Restoration of the Joint Line and Lateral Knee Pain in Patients Undergoing Total Knee Arthroplasty
}

\author{
Cetin Isik ${ }^{1}$, Uygar Dasar², Mesut Tahta ${ }^{3}$, Nurdan Cay, Ramazan Akmese5, Derya Isik6, \\ Murat Bozkurt1 \\ ${ }^{1}$ Department of Orthopaedics and Traumatology, Faculty of Medicine, Ankara Ataturk Training and Research \\ Hospital, Yildirim Beyazit University, Ankara, Turkey \\ ${ }^{2}$ Department of Orthopaedics and Traumatology, Faculty of Medicine, Karabuk Training and Research Hospital, \\ Karabuk University, Karabuk, Turkey \\ ${ }^{3}$ Department of Orthopaedics and Traumatology, Izmir Ataturk Training and Research Hospital, Izmir, Turkey \\ ${ }^{4}$ Department of Radiology, Faculty of Medicine, Ankara Ataturk Training and Research Hospital, Yildirim Beyazit \\ University, Ankara, Turkey \\ ${ }^{5}$ Department of Orthopaedics and Traumatology, Faculty of Medicine, Ankara University, Ankara, Turkey \\ ${ }^{6}$ Department of Family Medicine, Ankara Ataturk Training and Research Hospital, Ankara, Turkey \\ Email: ${ }^{*}$ ortdrcetin@hotmail.com
}

Received 17 May 2014; revised 17 June 2014; accepted 18 July 2014

Copyright () 2014 by authors and Scientific Research Publishing Inc.

This work is licensed under the Creative Commons Attribution International License (CC BY).

http://creativecommons.org/licenses/by/4.0/

(c) (i) Open Access

\begin{abstract}
Aim: In this study, the relationship between the change in the joint line and lateral knee pain was evaluated after primary total knee arthroplasty. Material and method: Between 2005 and 2012, patients who underwent primary total knee arthroplasty were included in the study. Patients having "cruciate retaining total knee arthroplasty" and with a change of the joint line $\geq 8 \mathrm{~mm}$ and patients having "posterior stabilized total knee arthroplasty" and with a change of the joint line $\geq 5$ $\mathrm{mm}$ were selected as the study group (group $1, \mathrm{n}=32$ ). A total of 47 patients having similar demographic characteristics with the study group and the joint line changing below the predetermined level or remaining unchanged were included as the control group (group 2). The 2 groups were compared according to the presence of knee pain, the localization and spread of the pain, iliotibial band, tension and pain in the patellar tendon and quadriceps tendon, front knee pain during squatting, VAS pain score, OBER test positivity, Knee Society knee and function score, and general patient satisfaction. Results: There were statistically significant more lateral knee pain $(p<0.001)$,
\end{abstract}

\footnotetext{
${ }^{*}$ Corresponding author.
}

How to cite this paper: Isik, C., et al. (2014) The Relationship between Incorrect Restoration of the Joint Line and Lateral Knee pain in Patients Undergoing Total Knee Arthroplasty. Open Journal of Rheumatology and Autoimmune Diseases, 4, 138-145. http://dx.doi.org/10.4236/ojra.2014.43020 
OBER test positivity $(p<0.001)$ and iliotibial band tension $(p<0.001)$ in group 1 compared to group 2 . However, there were no statistically significant differences between the 2 groups regarding rest pain $(p=0.855)$, pain during squatting $(p=0.761)$, exertional pain $(p=0.322)$, pain in the patellar tendon $(p=0.643)$ and quadriceps tendon $(p=0.873)$, Knee Society knee $(p=0.954)$ and function $(p=0.955)$ scores, and general satisfaction $(p=0.968)$. Conclusion: In total knee prosthesis operations, distal displacement of the joint line can result in lateral knee pain and iliotibial band tension. However, considering the results of total knee arthroplasty our findings have showed that this condition has no effect on knee functions and patient satisfaction.

\section{Keywords}

Joint Level, Knee Replacement Arthroplasty, Lateral Knee Pain

\section{Introduction}

Total knee arthroplasty (TKA) is one of the most successfully performed surgical procedures nowadays. TKA is forming an artificial joint, which the joint surfaces are covered by specially shaped metal and polyethylene. It is widely used in the treatment of excessively worn joint surfaces by osteoarthritis, trauma, age, obesity, rheumatologic disorders and previous cartilage or meniscus surgery. Although the number of patients who underwent surgical procedures has continuously increased in recent years, there is no similar rate of increase in patient satisfaction [1]. Unresolved knee pain after surgery is one of the most encountered reason of this dissatisfaction [2]. Too many studies have been performed about pain. In these studies, many factors such as age, sex, drug use, comorbidities, physiological factors, and knee scores have been evaluated [3] [4]. There are various methods including excision or preservation of the posterior cruciate ligament (PCL) although the anterior cruciate ligament (ACL) is routinely excised in TKA. As the tolerability border is different in prosthesis types including posterior cruciate ligament retaining total knee arthroplasty (CR-TKA) and posterior stabilized total knee arthroplasty (PS-TKA) types, incorrect restoration of the original joint line can cause primarily pain, instability, motion limitation, and joint stiffness [5].

In some patients, pain is in the form of lateral knee pain. There are publications reporting the significant correlation between iliotibial band tension and lateral knee pain. In addition, it has been reported that this condition can affect the scores and satisfaction after surgery [6] [7].

In the present study, patients were evaluated in terms of changes in the joint line after TKA. The relationship between the change in the joint line and lateral knee pain was investigated.

\section{Materials and Methods}

A total of 748 patient files containing primary total knee arthroplasty information were evaluated in the Department of Orthopedics and Traumatology, Ataturk Training and Research Hospital between November 2005 and June 2012. From these files, 39 containing information about distal displacement of the joint line $\geq 5 \mathrm{~mm}$ in cases with PS-TKA and $\geq 8 \mathrm{~mm}$ in cases with CR-TKA were selected. In the second elimination, 7 patient files containing data such as bilateral total knee arthroplasty, previously diagnosed iliotibial band syndrome, inflammatory arthritis, and gonarthrosis emerged after posttraumatic arthritis, valgus knees, varus knees $\geq 15^{\circ}$, and preoperative lateral knee pain were excluded from the evaluation. Remaining 32 patients were accepted as the study group (group 1). From excluded 709 patient files, 47 patients having similar characteristics with the study group were selected and accepted as the control group (group 2).

The mean age of the total population was 69.1 years (age range, 59 - 84 years) and the mean follow-up period was 48.5 months (range, 15 - 81 months). Forty seven patients were female and 32 were male. The right knee was operated in 44 patients and 35 patients had left knee surgery. Fifty three patients had CR-TKA and 26 had PS-TKA (Table 1 ).

Three different prosthesis brands with the combination of CR-PS TKA and mobile/fixed type insert were used in all patients. Mid-patellar longitudinal incision was used in all cases. For the determination of the joint line change, in postero-anterior knee graphs in full extension before and after surgery, distances between the tips of 
Table 1. Baseline characteristics of 2 study groups.

\begin{tabular}{ccccccccc}
\hline & $\begin{array}{c}\text { Mean age } \\
\text { (Year) }\end{array}$ & $\begin{array}{c}\text { Follow-up } \\
\text { period (Month) }\end{array}$ & $\begin{array}{c}\text { Sex } \\
\text { (F/M) }\end{array}$ & $\begin{array}{c}\text { Side } \\
\text { (Right/Left) }\end{array}$ & $\begin{array}{c}\text { CR-TKR + } \\
\text { mobile insert }\end{array}$ & $\begin{array}{c}\text { CR-TKR + } \\
\text { fixed insert }\end{array}$ & $\begin{array}{c}\text { PS-TKR + } \\
\text { mobile insert }\end{array}$ & $\begin{array}{c}\text { PS-TKR + } \\
\text { fixed insert }\end{array}$ \\
\hline Group 1 & 69.6 & 49.8 & $18 / 14$ & $19 / 13$ & 16 & 5 & 4 & 7 \\
Group 2 & 68.7 & 47.6 & $29 / 18$ & $25 / 22$ & 25 & 7 & 6 & 9 \\
\hline
\end{tabular}

the medial and lateral epicondyles and the distal femoral joint facet were measured separately (Figure 1 and Figure 2). Among the measurements performed from the both medial and lateral sides before and after surgery, observed change $\geq 5 \mathrm{~mm}$ for PS-TKA and $\geq 8 \mathrm{~mm}$ for CR-TKA were accepted as positive for the joint line change and included in group 1 . Cases having a change above the predetermined values only in one measurement (medial or lateral) were excluded. Because the distal femoral incision could be performed with inappropriate varus-valgus degrees and this condition can give incorrect information about the joint line change. The measurements were repeated 3 times by 1 observer taking into account intra-observer variability.

The 2 groups were compared regarding knee pain, localization and spread of the pain (in iliotibial band, patellar tendon and quadriceps tendon), the response to anti-inflammatory drugs, the relation to rest and exercise (pain increase with squatting), and general satisfaction. For detecting pain in the patellar tendon, the middle point between the tibial tuberosity and the distal tip of the patella was palpated. For detecting pain in the quadriceps tendon, approximately $2 \mathrm{~cm}$ proximal portion of the patellar tip was palpated. The most clearly palpated portion of the iliotibial band over the distal-lateral surface of the thigh was used to detect pain in the iliotibial band. VAS score was used for detecting pain levels.VAS is a psychometric response scale which can be used in questionnaires. It is a measurement instrument for subjective characteristics or attitudes that cannot be directly measured.

In both groups, the tension in the iliotibial band, patellar tendon and quadriceps tendon was evaluated by palpation in comparison with contralateral side. Because of the effect of incorrect restoration of the joint line on extension distance, the tension was evaluated in full extension of the knee and hip. Both groups were compared regarding OBER test, stability, joint range of mobility, and Knee Society (knee and function) Scores (KSS). OBER test is used for test to evaluate a tight, contracted or inflamed iliotibial tract; the patient lies on the uninvolved side and the involved hip is abducted by the examiner as the knee is flexed to 90 degrees; the hip is allowed to adduct passively; the degree of abduction or the production of pain al found on. For continues and categorical variables Student's $t$ test and $x^{2}$ test were used, respectively. Cronbach-Alpha test was used for intraobserver variability.

\section{Results}

The reliability rate of measurements for determining the change in the joint line of patients in both groups was found to be high $(p<0.001)$.

There were no statistically significant differences regarding rest pain $(p=0.855)$, exertional pain $(p=0.322)$ and pain during squatting ( $p=0.761$ ) between the 2 groups. The mean iliotibial band pain VAS score was 4.7 in group 1 compared to 2.2 in group $2(p<0.001)$. In addition, there was no statistically significant difference regarding quadriceps tendon pain $(p=0.873)$ (Table 2).

The iliotibial band tension was detected in $67 \%$ of group 1 patients compared to $17 \%$ of group 2 patients ( $p<$ 0.001). OBER test was positive in $77 \%$ of group 1 patients compared to $19 \%$ of group 2 patients $(p<0.001)$. There were no statistically significant differences between the 2 groups regarding patellar tendon tension $(p=$ $0.679)$ and quadriceps tendon tension $(p=0.832)$ (Table 3$)$.

The requirement rate for intraoperative lateral retinacular relaxation was 18\% (6 patients) in group 1 compared to $11 \%$ (5 patients) in group 2. This finding supports the notion that lateral knee pain detected as significantly higher in group 1 was not from the patellar source.

In group 1, $21(65 \%)$ patients had flexion contracture of $5^{\circ}-10^{\circ}$ and $11(35 \%)$ had flexion contracture of 10 $15^{\circ}$. There was no patient having flexion contracture $>15^{\circ}$. In group 2, 32 (68\%) patients had flexion contracture of $5^{\circ}-10^{\circ}$ and $13(27 \%)$ had flexion contracture of $10^{\circ}-15^{\circ}$ (Table 4). Knee extension of 2 (5\%) patients having passive flexion contracture of $16^{\circ}-20^{\circ}$ was achieved up to $10^{\circ}$ by manipulation. There was no statistically significant difference between the 2 groups regarding flexion contracture. 


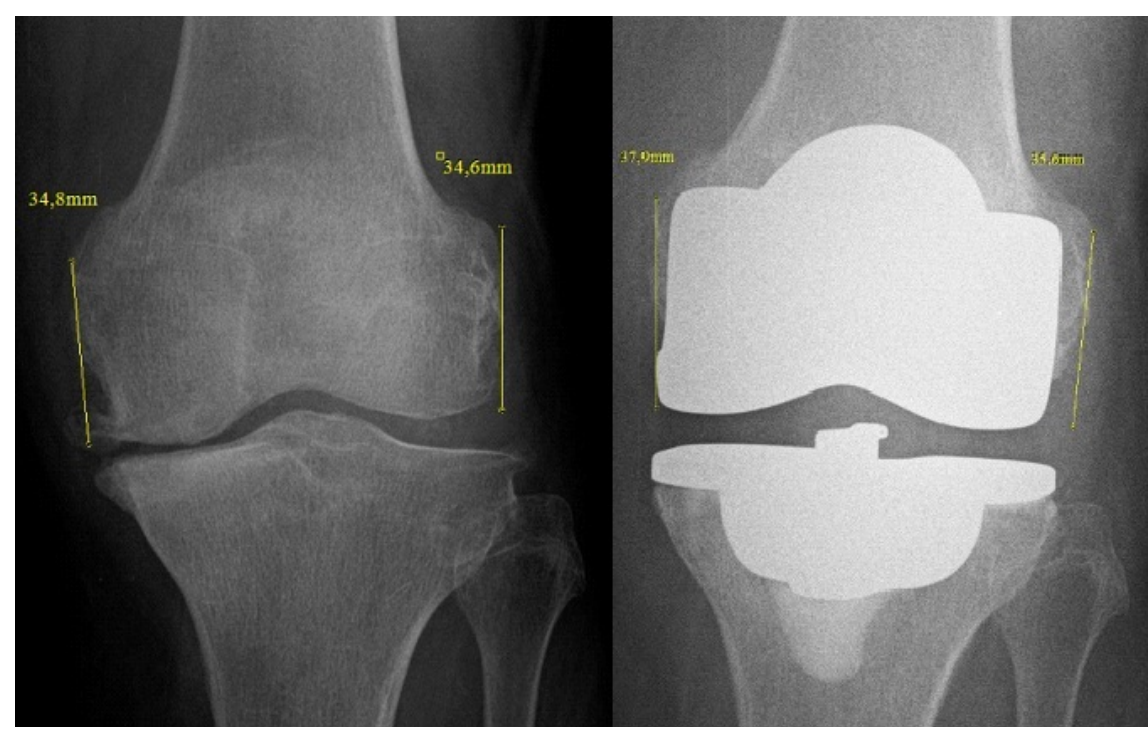

Figure 1. The measurements on antero-posterior graph from a patient without a change in the joint line.

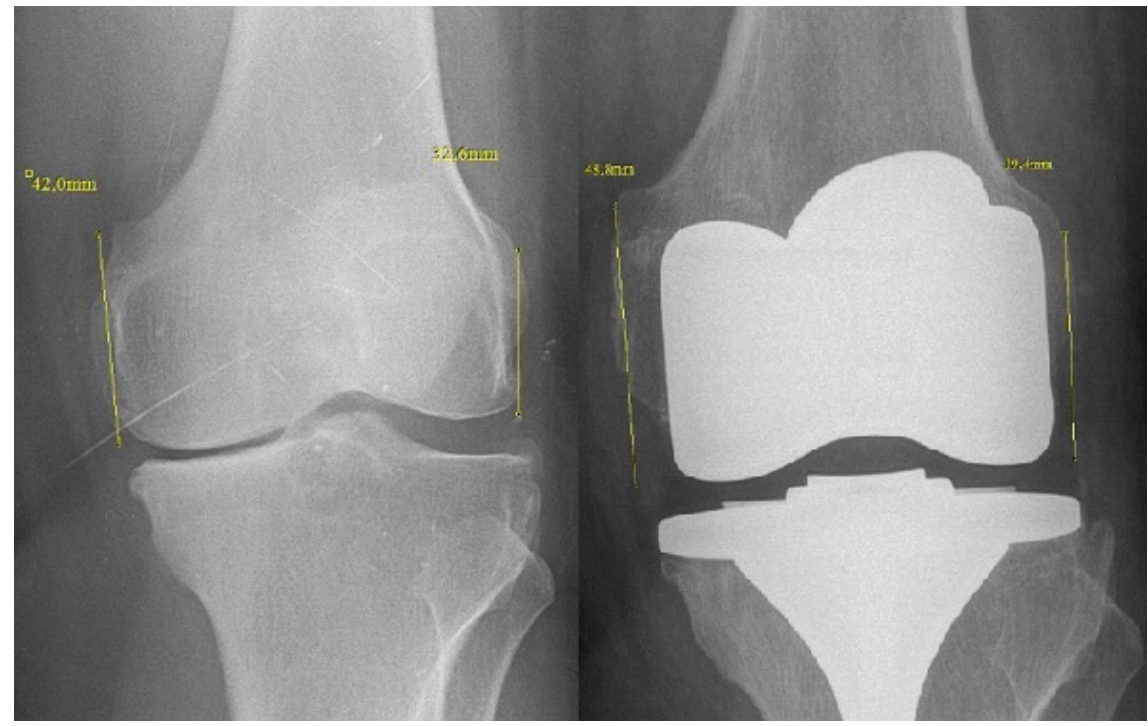

Figure 2. The measurements on antero-posterior graph from a patient with a change in the joint line.

Table 2. The mean VAS scores of 2 study groups.

\begin{tabular}{lcccccccc}
\hline & Rest pain & Exertional pain & ITB pain & PT pain & Quadriceps pain & Squatting pain & General satisfaction \\
\hline Group 1 & 2.2 & 3.1 & 4.7 & 2.3 & 3.6 & 5.8 & 3.3 \\
Group 2 & 2.1 & 1.9 & 2.2 & 1.5 & 5.2 & 6.8 & 3.4 \\
\hline
\end{tabular}

Table 3. Physical findings and mean scores of 2 study groups.

\begin{tabular}{ccccccc}
\hline & ITB tension (+) & PT tension (+) & Quadriceps tension (+) & OBER (+) & KS knee score & KS function score \\
\hline Group 1 & $67 \%$ & $21 \%$ & $9 \%$ & $77 \%$ & 86 & 82 \\
Group 2 & $17 \%$ & $25 \%$ & $12 \%$ & $19 \%$ & 89 & 90 \\
\hline
\end{tabular}


Table 4. Flexion contracture distribution between 2 groups.

\begin{tabular}{cccc} 
& $\mathbf{5}^{\circ}-\mathbf{1 0}^{\circ}$ & $\mathbf{1 6}^{\circ}-\mathbf{2 0}^{\circ}$ & $>\mathbf{2 0}^{\circ}$ \\
\hline Group 1 & $21(65 \%)$ & $11(35 \%)$ & - \\
Group 2 & $32(68 \%)$ & $13(27 \%)$ & $2(5 \%)$ \\
\hline
\end{tabular}

In group 1, 29 (91\%) patients had varus-valgus $<5^{\circ}, 1(3 \%)$ had $6^{\circ}$ varus and $2(6 \%)$ had $8^{\circ}$ valgus on anteroposterior graph. In group 2, 43 (91\%) patients had varus-valgus $<5^{\circ}, 2$ (4\%) had $6^{\circ}$ valgus, 1 (2.5\%) had $9^{\circ}$ valgus, and $1(2.5 \%)$ had $7^{\circ}$ varus on antero-posterior graph (Table 5). There were no statistically significant differences between the 2 groups regarding varus/valgus deformities. There was a homogenous distribution regarding sequence between the groups. This finding rules out the probability of postoperative lower extremity sequence as a factor that will play a role on lateral knee pain and tension.

There were no statistically significant differences regarding Knee Society knee $(p=0.954)$ and function $(p=$ 0.955) score between the 2 groups. In group 1, 82\% of patients had an intermediate or high satisfaction level compared to $18 \%$ with low satisfaction or dissatisfaction level. In group 2, $85 \%$ of patients had an intermediate or high satisfaction level compared to $15 \%$ with low satisfaction or dissatisfaction level. No statistically significant difference was found regarding satisfaction between the 2 groups.

\section{Discussion}

Unresolved knee pain seen in approximately $20 \%$ of patients after total knee arthroplasty is a serious complication which can cause medical, social and legal problems [8]. Pain related to patella-femoral joint and extensor mechanism is frequently seen and this condition is in the first place when TKA revision indications of non-infectious cause are handled. For an effective treatment, pathology causing the pain should be diagnosed [9].

Although studies related to pain after surgery have been found, no sufficient literature studies about localization of pain and related probable pathologies are present. In addition to this, Pandher et al. presented a case with posterolateral knee pain after total knee arthroplasty. They linked this pain to inflammation of the biceps femoris [10]. Bozkurt et al. demonstrated that there was a strong correlation between lateral knee pain and lateral tension. They also reported that lateral hamstring and/or iliotibial band tensions were important reasons of lateral knee pain [6]. Similarly, Can et al. reported that anterior knee pain and lateral tension after total knee arthroplasty significantly and negatively influenced the clinical results [7].

VAS score is an extensively used parameter for determining pain after surgery. In a study of primary total knee arthroplasty by Brander et al., knee pain of cases was determined using VAS score and at the end of the 1-year follow-up period, the score was 1.7 [11]. Heck et al. also detected similar results using the same method [12]. Bullens et al. performed a similar study with a follow-up period of 5 years and found that VAS score as knee pain was 1.2 [13]. Pleogmakers et al. evaluated the designs of total knee prostheses in their study. They also used VAS score together with many parameters for knee pain and had reported that the mean VAS score for knee pain of patients after surgery was 3 [14].

In our study, VAS score was used for determining of knee pain of patients. During rest, pain was found to be 2.2 in group 1 compared to 2.1 in group 2. Exertional pain was, however, 3.1 in group 1 compared to 1.9 in group 2 although this difference was statistically insignificant. This higher score could explain pain formation resulted from repetitive flexion-extension movements of the iliotibial band. In addition, significantly higher score of the iliotibial band pain and tension in group 1 and the higher prevalence of patients with OBER test positivity in group 1 supported this idea.

Anatomic restoration of the joint line level during surgery is one of the key factors to provide appropriate connective tissue balance and normal knee kinematics and to obtain satisfactory results after TKA [15]. The majority of studies about this issue have focused on revision TKA more than primary TKA [16] [17]. In our present study, we demonstrated that correct restoration of the joint line in primary total knee arthroplasty was one of the necessary factors for a good clinical result.

There is no standard anatomical measurement technique to use correct identification of the joint line. The distance between anatomical points such as adductor tubercle, medial epicondyle, lateral epicondyle and distal femoral articular facet may be chosen for determining the joint line. The distance between the proximal part of the fibular head or tibial tubercle and proximal tibial articular facet may also be preferred for determining the joint 
Table 5. Varus/valgus deformity distribution between 2 groups.

\begin{tabular}{ccc} 
& $\mathbf{0}^{\circ}-5^{\circ}$ & $5^{\circ}-\mathbf{1 1}^{\circ}$ \\
\hline Group 1 & $29(91 \%)$ & $3(9 \%)$ \\
Group 2 & $43(91 \%)$ & $4(9 \%)$ \\
\hline
\end{tabular}

line [18] [19]. In our study, to determine the joint line, we measured distances between the medial and lateral epicondyles and the distal femoral joint surface. To exclude the probability of inappropriate varus or valgus positional incision of the distal femur during surgery, when the 2 measured distances were above the predetermined threshold values it was accepted as a significant change of the joint line.

If the joint line restoration is incorrect after primary or revision TKA, surgical result cannot be good enough than expected. It has been reported that incorrect restoration of the joint line can cause not only instability but also knee pain and motion limitation [20]. In a study by Partington et al. [21] they reported that a change $\geq 8 \mathrm{~mm}$ in the joint line significantly decreased KSS. In a cadaveric study, Martin and Whiteside demonstrated that proximal displacement of the joint line $5 \mathrm{~mm}$ anteriorly and proximally could result in midflexion instability of the knee [19]. Konig et al. showed that a $10-\mathrm{mm}$ change in the joint line increased patella-femoral forces by $60 \%$ [22]. In addition to these findings, $\geq 8 \mathrm{~mm}$ increase or decrease of the joint line decreased the range of motion by approximately $14^{\circ}$ and according to lower changes from these levels knee functional scores decreased by 11 points [23]. In a cadaveric study by Fornalski et al. $\geq 4 \mathrm{~mm}$ change in the joint level significantly increased the patella-femoral contact forces. They also reported that especially $\geq 8 \mathrm{~mm}$ change resulted in abnormal loadings. In contrary to this, as defended by Scuderi and Insall [24], in a retrospective study Selvarajah et al. [25] defended that up to $10 \mathrm{~mm}$, a change in the joint line had no effect on any clinical result.

In our study, with regard to KSS, there was no significant difference between the joint line changing and unchanging groups. In other words, incorrect restoration of the joint line did not affect the clinical score. KSS had a wide field of use in orthopedic literature. It had a high sensitivity to evaluate the patient after primary or revision knee prosthesis [26]. It had also been reported that intra-observer and inter-observer reliability of the test were significantly high. We showed that lateral knee pain and tension in lateral part of the knee significantly increased in patients having change in the joint line. In addition, OBER test positivity rate significantly increased in joint line changing group. According to our results, a pre-specified change of the joint line had no effect on general satisfaction and knee scoring but this condition could cause some specific complaints such as lateral knee pain and lateral tension.

There are some limitations of our study. First, the study has a retrospective nature. Second, conversion of pain has the characteristic of subjectivity to numeric values. Third, some parameters such as tension tests have no quantitative values and they are presented as absent or present. Further studies including examined criteria and new scoring systems are needed to achieve more objective results.

Consequently as in all surgical procedures, total knee arthroplasty has important and critical steps such as correct restoration of the joint line. In our study, we demonstrated that distal displacement of the joint line had no effect on functional scores, patient satisfaction and anterior knee pain. In addition, founded significant relationship between the change in the joint line and the lateral knee pain and tension was thought to be a condition that should be considered.

\section{References}

[1] Ritter, M.A. (2009) The Anatomical Graduated Component Total Knee Replacement: A Long-Term Evaluation with 20 Year Survival Analysis. The Bone \& Joint Journal, 91, 745-749. http://dx.doi.org/10.1302/0301-620X.91B6.21854

[2] Hawker, G., Wright, J., Coyte, P., Paul, J., Dittus, R., Croxford, R., Katz, B., Bombardier, C., Heck, D., Freund, D., et al. (1998) Health-Related Quality of Life after Knee Replacement. The Bone \& Joint Journal, 80, 163-173.

[3] Baker, P.N., van der Meulen, J.H., Lewsey, J. and Gregg, P.J. (2007) The Role of Pain and Function in Determining Patient Satisfaction after Total Knee Replacement. Data from the National Joint Registry for England and Wales. The Bone \& Joint Journal, 89,893-900. http://dx.doi.org/10.1302/0301-620X.89B7.19091

[4] Bourne, R.B., Chesworth, B.M., Davis, A.M., Mahomed, N.N. and Charron, K.D. (2010) Patient Satisfaction after Total Knee Arthroplasty: Who Is Satisfied and Who Is Not? Clinical Orthopaedics and Related Research, 468, 57-63. http://dx.doi.org/10.1007/s11999-009-1119-9

[5] Wyss, T.F., Schuster, A.J., Munger, P., et al. (2006) Does Total Knee Joint Replacement with the Soft Tissue Balanc- 
ing Surgical Technique Maintain the Natural Joint Line? Archives of Orthopaedic and Trauma Surgery, 7, 480-486. http://dx.doi.org/10.1007/s00402-006-0171-0

[6] Bozkurt, M., Can, F., Erden, Z. and Demirkale, I. (2003) The Influence of Lateral Tightness on Lateral Knee Pain. The Pain Clinic, 16, 1-6.

[7] Can, F., Bozkurt, M., Erden, Z. and Aygul, V. (2005) The Influence of Anterior Knee Pain and Lateral Tightness on Knee Function in Total Knee Arthroplasty. Physical Therapy, Abstract.

[8] Bonnin, M.P., Basiglini, L. and Archbold, H.A. (2011) What Are the Factors of Residual Pain after Uncomplicated TKA? Knee Surgery, Sports Traumatology, Arthroscopy, 19, 1411-1417.

[9] Sharkey, P.F., Hozack, W.J., Rothman, R.H., Shastri, S. and Jacoby, S.M. (2002) Why Are Total Knee Arthroplasties Failing Today? Clinical Orthopaedics and Related Research, 404, 7-13. http://dx.doi.org/10.1097/00003086-200211000-00003

[10] Luyckx, L., Luyckx, T., Bellemans, J. and Victor, J. (2010) Iliotibial Band Traction Syndrome in Guided Motion TKA A New Clinical Entity after TKA. Acta Orthopaeligdica Belgica, 76, 507-512

[11] Brander, V.A., Stulberg, S.D., Adams, A.D., Harden, R.N., Bruehl, S., Stanos, S.P. and Houle, T. (2003) Predicting Total Knee Replacement Pain: A Prospective, Observational Study. Clinical Orthopaedics and Related Research, 416, 27-36.

[12] Heck, D.A., Robinson, R.L., Partridge, C.M., Lubitz, R.M. and Freund, D.A. (1998) Patient Outcomes after Knee Replacement. Clinical Orthopaedics and Related Research, 356, 93-110. http://dx.doi.org/10.1097/00003086-199811000-00015

[13] Bullens, P.H., Van Loon, C.J., De Waal Malefijt, M.C., Laan, R.F. and Veth, RP. (2001) Patient Satisfaction after Total Knee Arthroplasty: A Comparison between Subjective and Objective Outcome Assessments. The Journal of Arthroplasty, 1, 740-747. http://dx.doi.org/10.1054/arth.2001.23922

[14] Ploegmakers, M.J.M., Ginsel, B., Meijerink, H.J., de Rooy, J.V., de Waal Malefijt, M.J., Verdonschot, N. and Banks, S.A. (2010) Physical Examination and in Vivo Kinematics in Two Posterior Cruciate Ligament Retaining Total Knee Arthroplasty Designs. The Knee, 17, 204-209. http://dx.doi.org/10.1016/j.knee.2009.09.005

[15] Hofmann, A.A., Kurtin, S.M., Lyons, S., et al. (2006) Clinical and Radiographical Analysis of Accurate Restoration of the Joint Line in Revision Total Knee Arthroplasty. The Journal of Arthroplasty, 21, 1154-1162. http://dx.doi.org/10.1016/j.arth.2005.10.026

[16] Mason, M., Belisle, A., Bonutti, P., Kolisek, F.R., Malkani, A. and Masini, M. (2006) An Accurate and Reproducible Method for Locating the Joint Line during a Revision Total Knee Arthroplasty. Journal of Arthroplasty, 21, 1147-1153. http://dx.doi.org/10.1016/j.arth.2005.08.028

[17] Porteous, A.J., Hassaballa, M.A. and Newman, J.H. (2008) Does the Joint Line Matter in Revision Total Knee Replacement? Journal of Bone and Joint Surgery-British Volume, 90, 879-884. http://dx.doi.org/10.1302/0301-620X.90B7.20566

[18] Romero, J., Seifert, B., Reinhardt, O., Ziegler, O. and Kessler, O. (2010) A Useful Radiologic Method for Preoperative Joint-Line Determination in Revision Total Knee Arthroplasty. Clinical Orthopaedics and Related Research, 468, 1279-1283. http://dx.doi.org/10.1007/s11999-009-1114-1

[19] Martin, J.W. and Whiteside, L.A. (1990) The Influence of Joint Line Position on Knee Stability after Condylar Knee Arthroplasty. Clinical Orthopaedics and Related Research, 259, 146-156.

[20] Figgie, H.E., Goldberg, V.M., Heiple, K.G., Moller, H.S. and Gordon, N.H. (1986) The Influence of Tibial-Patellofemoral Location on Function of the Knee in Patients with the Posterior Stabilized Condylar Knee Prosthesis. Journal of Bone and Joint Surgery, 68, 1035-1040.

[21] Partington, P.F., Sawhney, J., Rorabeck, C.H., Barrack, R.L. and Moore, J. (1999) Joint Line Restoration after Revision Total Knee Arthroplasty. Clinical Orthopaedics and Related Research, 365, 165-171.

[22] Konig, C., Sharenkov, A., Matziolis, G., Taylor, W.R., Perka, C., Duda, G.N. and Heller, M.O. (2009) Joint Line Elevation in Revision TKA Leads to Increased Patellofemoral Contact Forces. Journal of Orthopaedic Research, 28, 1-5. http://dx.doi.org/10.1002/jor.20952

[23] National Center for Health Statistics (1999) Arthritis. American Academy and American Association of Orthopaedic Surgeons Bulletin, 47, 14.

[24] Scuderi, G.R. and Insall, J.N. (1989) The Posterior Stabilized Knee Prosthesis. Orthopedic Clinics of North America, 20, 71.

[25] Selvarajah, E. and Hooper, G. (2009) Restoration of the Joint Line in Total Knee Arthroplasty. The Journal of Arthroplasty, 24, 1099-1102. http://dx.doi.org/10.1016/j.arth.2008.06.030

[26] Ewald, F.C. (1989) The Knee Society Total Knee Arthroplasty Roentgenographic Evaluation and Scoring System. Clinical Orthopaedics and Related Research, 248, 9-12. 


\section{Abbreviations}

TKA: Total knee arthroplasty.

CR-TKA: Posterior cruciate ligament retaining total knee arthroplasty.

PS-TKA: Posterior stabilized total knee arthroplasty.

VAS: Visual analogue scale.

Ober Test: Test to evaluate a tight, contracted or inflamed ilotibial tract.

KSS: Knee society score. 
Scientific Research Publishing (SCIRP) is one of the largest Open Access journal publishers. It is currently publishing more than 200 open access, online, peer-reviewed journals covering a wide range of academic disciplines. SCIRP serves the worldwide academic communities and contributes to the progress and application of science with its publication.

Other selected journals from SCIRP are listed as below. Submit your manuscript to us via either submit@scirp.org or Online Submission Portal.
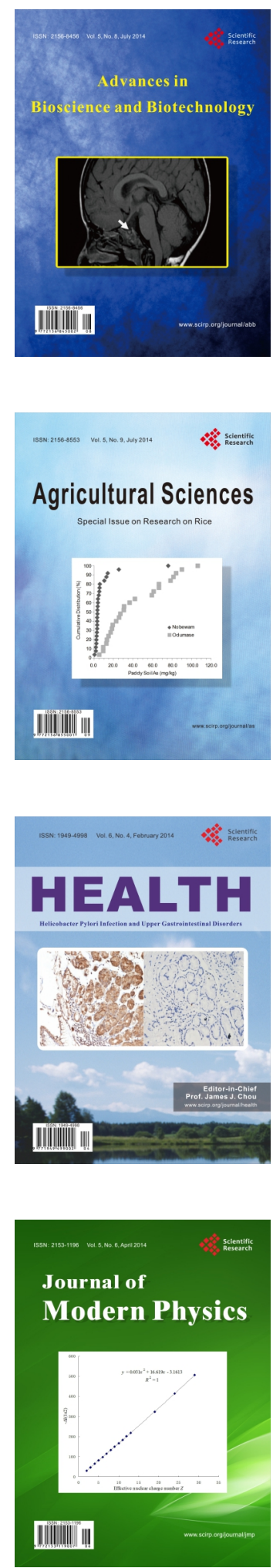
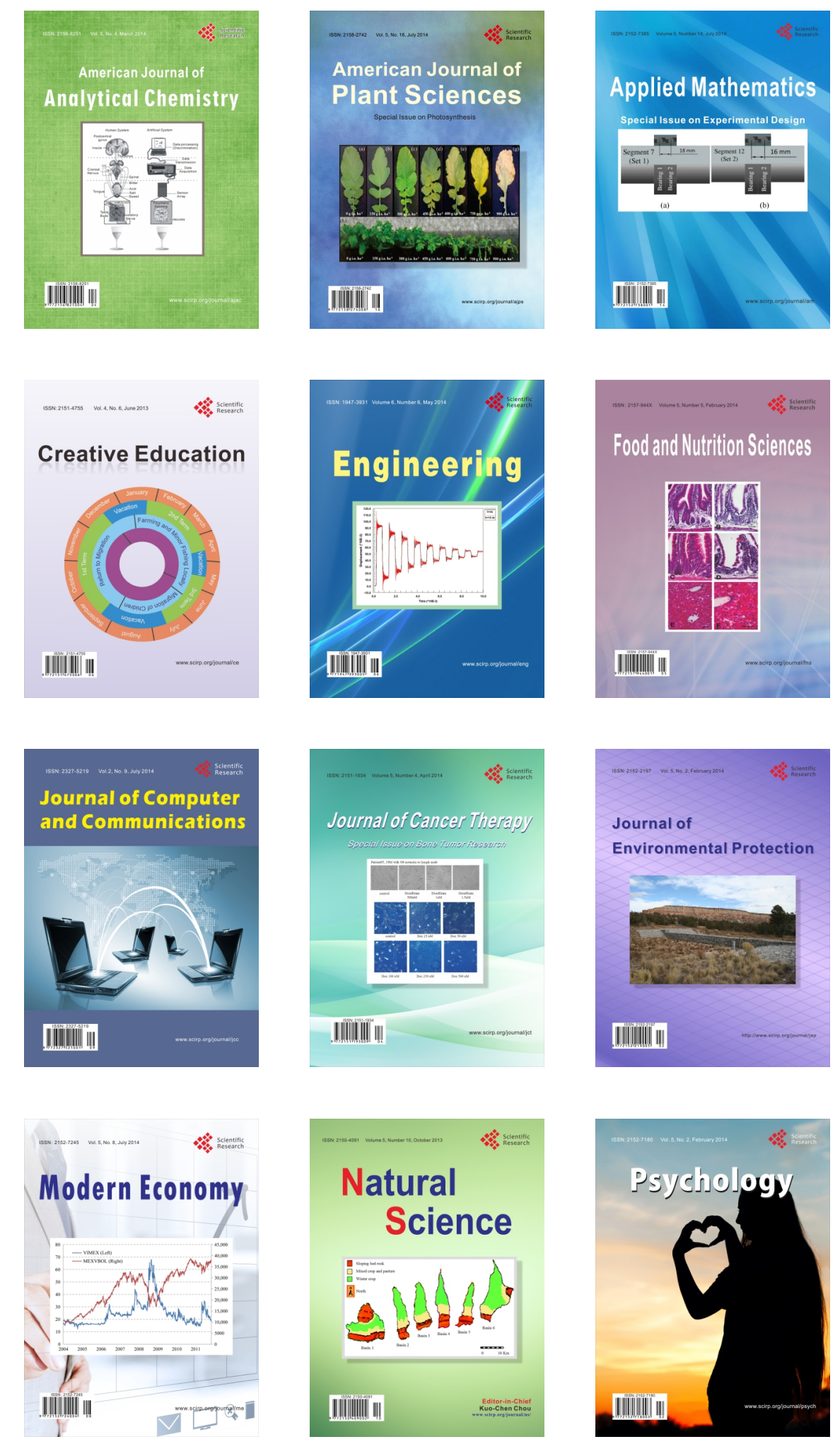